\title{
Global Geometric Distortion Correction in Images
}

\author{
Mohammad Awrangjeb, Manzur Murshed, and Guojun Lu \\ Gippsland School of Information Technology, Monash University, Churchill Vic 3842, Australia \\ \{Mohammad.Awrangjeb, Manzur.Murshed, Guojun.Lu\}@infotech.monash.edu.au
}

\begin{abstract}
The performance of existing copyright protection schemes is questionable due to their vulnerability to geometric transformations. Though a few of them can resist global geometric transformations like rotation and scaling attacks, most of them are vulnerable to rotation-scale and cropping attacks. This paper presents a novel geometric distortion correction scheme robust to global geometric transformations. It restores an attacked image to its approximate original by reversing the attack using the invariant centroid and geometric moments of the image. Experimental results show the effectiveness of the proposed scheme.
\end{abstract}

Keywords - copyright protection; geometric transformation; rotation; scaling; invariant centroid; moments

Topic area-multimeida security.

\section{INTRODUCTION}

Due to ever-growing expansion of using digital multimedia and the Internet, the problem of ownership protection of digital media has become increasingly important. Although significant progress has been made, surviving of the watermark in geometric attacks is still an active research area. Such attacks are easy to implement, but can make most of the existing watermarking schemes ineffective. Geometric transformations can be decomposed into two classes: global transformations such as rotation, scaling, and translation (RST) and local transformations like local bending attacks. A lot of schemes [1-3, 5-20] have been proposed to combat both types of transformations; nonetheless, the problem of resisting geometric attacks has been unsolved yet because of their high computational complexity, implementation difficulties, and poor performance. In addition, many of the existing schemes are vulnerable to rotation-scale and cropping attacks.

The detection or extraction of the watermark requires a synchronization step to locate the embedded mark in the content. However, geometric transformations of the watermarked content create synchronization problem in the watermark detection or extraction phase. There are many synchronizing schemes to combat geometric attacks in the literature $[1-3,5-20]$. They may be divided into following seven classes.

Alghoniemy and Tewfik proposed an exhaustive search scheme [1] that transformed the test image in every possible way in order to recover synchronization based on a training sequence against rotation and scaling attacks. However, the time complexity of exhaustive search increases exponentially with the increase in search space i.e., the number of attacks.
Periodical sequence-based schemes add a sequence number periodically during the embedding process. This redundant information can be used latter to localize the position of the watermark and to improve the detection phase. Hartung et al. [2] applied a periodic insertion for synchronization and correlated the mark using a fourdimensional sliding correlator.

Template-based schemes identify the transformation by retrieving artificially embedded references known as templates. They embed templates in addition to the watermark reducing both the image fidelity and the embedding capacity. Pereira and Pun [3] localized templates in a ring corresponding to the middle frequencies of the spectrum. Templates were generated by increasing the magnitude of the selected coefficients and creating a local peak. Though template insertion-based schemes address synchronization after global and local geometric attacks, templates can easily be erased since they usually represent peaks in the transform domain [4]. A serious problem arises when many watermarked images share a common template easing collusion-based detection of the template and posing security threats to the template itself.

Invariant transform-based schemes embed the watermark in an invariant domain like Fourier-Mellin transform (FMT). The scheme in [5] was theoretically effective but difficult to implement due to poor interpolation accuracy during log-polar and inverse log-polar mapping (LPM, ILPM). Lin et al. [6] proposed an improvement reducing implementation difficulties; however, it suffered from frequency localization problem. Kim et al. [7] removed this problem by reordering and modifying FMT function blocks and using the invariant centroid (IC). However, they are still vulnerable to cropping and computationally expensive. Moreover, non-uniform sampling grid of the LPM causes aliasing.

Non-blind schemes use the original image in order to detect the mark by identifying the geometrical transformation applied on the image. Dong et al. [8] used a deformable mesh model to correct the geometric distortion. These schemes are effective for local distortions, but an extension to global affine transformations is computationally demanding [9].

Image normalization-based schemes use invariant geometric [10-11] or Zernike [12] moments to transfer the image into a standard form. Dong et al. [10] embedded multibit watermark while Alghoniemy and Tewfik [11] embedded 1-bit watermark only. However, image normalization is highly sensitive to cropping and computationally expensive if highorder moments are exploited. 
Content-based schemes belong to the second-generation watermarking schemes defined by Kutter et al. [13]. As the image content represents an invariant reference to geometrical transformations, these schemes locate the mark by linking with image semantics. For feature extraction they used Harris corner and edge detector [9, 19-20], Mexican Hat wavelet scale interaction model $[13,15]$, wavelet maxima [14], scaleinvariant feature transform [16], image segmentation [17], and scale-space representation of the image [18][20]. However, their effectiveness was limited. For example, the scheme in [14] was robust to small rotation and upscaling, but was severely weak against downscaling due to inherent data loss accompanied with the down sampling process. Color image segmentation algorithms [17] were fragile to downscaling and aspect-ratio change [21]. Though Harris corner detector extracts more robust features than Mexican Hat wavelet, the former is sensitive to blurring and cropping [16]. Combination of different schemes was also proposed in the literature to improve robustness. For instance, Tang and Hang [15] combined image normalization, periodical sequence, and content-based schemes; nevertheless, this combined scheme was sensitive to scaling and cropping [16].

Some common problems of abovementioned schemes are their low robustness against rotation-scale and cropping. In fact, FMT-based schemes are not capable of distinguishing between a resizing due to scaling and one due to cropping, since LPM is always sampled with the same number of points; image normalization-based schemes are highly sensitive to cropping; and content-based schemes are largely dependent on the accuracy of involved feature point detectors which are sensitive to cropping. Though exhaustive search technique outperforms template-based technique if the search space increases polynomially [22], the former is time-consuming.

The IC of an image can be detected under many RST attacks, even if the image is moderately cropped [7]. This paper presents a computationally inexpensive content-based geometric distortion correction scheme based on the IC and low order geometric moments and using general knowledge of geometric transformations. The proposed scheme can correct global geometric transformations like RST and their combinations e.g., rotation-scale attack. In addition, it is also robust to image cropping and flipping attacks.

\section{PRoposed SCHEME}

Geometric moments have been extensively explored in [11-12] with a view to cope with geometric transformations. Kim et al. [7] proposed a geometric moment-based iterative procedure for finding the IC of an image to achieve translation invariance. In section II.A and II.B, we will review them in order to make the paper self-contained. Then we will present the proposed geometric transformation correction scheme based on the IC and geometric moments and using general knowledge of geometric transformations in sections II.C and II.D. In section II.E, we will discuss how a global geometric attacked image can be restored to its approximate original.

\section{A. Geometric Moments}

Consider a gray-scale image $I$ of size $w_{I} \times h_{I}$, where $1 \leq \mathrm{x}$ $\leq w_{I}$ and $1 \leq y \leq h_{I}$. Define the geometric moments $m_{p, q}$ of $I$ as

$$
m_{p, q}=\sum_{x} \sum_{y} x^{p} y^{q} I(x, y)
$$

and the central moments $\mu_{p, q}$ as

$$
\mu_{p, q}=\sum_{x} \sum_{y}(x-\bar{x})^{p}(y-\bar{y})^{q} I(x, y),
$$

where $(\bar{x}, \bar{y})$ is the centroid of the image defined as

$$
\bar{x}=\frac{m_{1,0}}{m_{0,0}}, \bar{y}=\frac{m_{0,1}}{m_{0,0}} .
$$

The $x$ and $y$-directions in the above equations change when the image is rotated and scaled. In order to estimate these directions, we use the principal axis concept applied to the binary image [11]. The correction angle $\theta_{c}$ can be expressed as

$$
\theta_{c}=\frac{1}{2} \tan ^{-1} \frac{2 \mu_{1,1}}{\mu_{2,0}-\mu_{0,2}} .
$$

Again define the geometric moments computed from the center $\left(x_{0}, y_{0}\right)$ of the image as

$$
\tilde{\mu}_{p, q}=\sum_{x y} \sum_{y}\left(x-x_{0}\right)^{p}\left(y-y_{0}\right)^{q} I(x, y) .
$$

Note that $I(x, y)$ denotes the pixel value at position $(x, y)$ in image $I$.

\section{B. Invariant Centroid}

In conventional feature point techniques, it is difficult to find an invariant point that is not changed by geometric transformations, image processing, and compression. Kim et al. [7] proposed an iterative method (see Fig. 1) to find out the IC $C=\left(c_{x}, c_{y}\right)$ in an image, where $\left(c_{x}, c_{y}\right) \equiv(\bar{x}, \bar{y})$ as defined in (3).

The method first passes the image through a Gaussian low pass filter which reduces the effect of waveform attacks like JPEG lossy compression, noise addition etc. The initial centroid $C_{0}$ is calculated over the entire image using (3). In order to reduce the effect due to both cropping by geometric

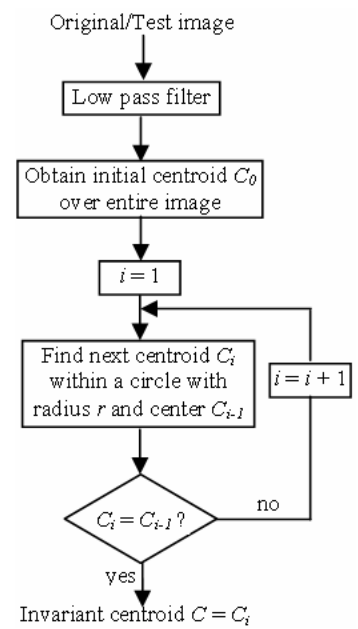

Fig. 1: Finding invariant centroid of an image 
attacks and change of pixel values by waveform attacks, this method then uses a circular region centered at the centroid $C$ with radius $r$. Note that the value of $C$ in this iterative method converges after a few iterations. However, for images full of detail texture and regular pattern and brightness change such as chessboard, the IC may not be converged to a unique point. Nevertheless, real world images, in general, do not have such compositions [7].

\section{Global Geometric Distortion Correction}

The original image is not required for distortion corrections. However, it is assumed that some of its information like its size $w_{I} \times h_{I}$, IC $C$, radius $r$, and signs of $\widetilde{\mu}_{1,0}$ and $\tilde{\mu}_{0,1}$ are provided.

1) Flipping: Flipping can be a part of an RST attack. We achieve flipping invariance using signs of $\tilde{\mu}_{1,0}$ and $\tilde{\mu}_{0,1}$ as they change their signs with flipping around $y$ and $x$-axis respectively [11].

2) Cropping: The cropping invariance is achieved using $C$ as it is invariant to the center $\left(x_{0}, y_{0}\right)$. In both symmetric and asymmetric cropping attacks, the IC in the attacked image can be found at the same position as the original IC.

3) Translation: Cropping and translation attacks are similar but in translation the original size of the image is preserved. In translation attack, the IC shifts as the same amount as the centre. Let the IC in the attacked image be $C_{t}=\left(c_{x t}, c_{y t}\right)$ due to a translation of the center $\left(x_{0}, y_{0}\right)$ to $\left(t_{x}, t_{y}\right)$. Consequently, we can find the translation amount as

$$
t_{x}=c_{x t}-c_{x} \text { and } t_{y}=c_{y t}-c_{y} .
$$

4) Rotation-only and Rotation-crop: In rotation attack, the rotated image may or may not be cropped. If $I$ is rotated by an angle $\theta_{r}$, the IC is also rotated by the same angle, irrespective of the cropping after rotation. Let the IC of the rotated image be $C_{r}=\left(c_{x r}, c_{y r}\right)$. Then $\theta_{r}$ can be calculated as

$$
\theta_{r}=\alpha_{r}-\alpha
$$

where $\alpha_{r}$ and $\alpha$ are angular distances of $C_{r}$ and $C$ calculated as

$$
\alpha_{r}=\tan ^{-1}\left(\frac{c_{y r}}{c_{x r}}\right) \text { and } \alpha=\tan ^{-1}\left(\frac{c_{y}}{c_{x}}\right) .
$$

5) Scale and scaleup-crop: In scale attack, if $I$ is scaled by a factor $s$ in both $x$ and $y$-directions, the linear distance of the IC from the center of the image changes by the same factor; however, its angular distance does not change. A cropping can be done after scaleup. Let the IC of the scaled image be $C_{s}=\left(c_{x s}, c_{y s}\right)$. Then $s$ can be calculated as

$$
s=\frac{R_{S}}{R},
$$

where $R_{s}$ and $R$ are linear distances of $C_{s}$ and $C$ calculated as

$$
R_{s}=\sqrt{c_{x s}^{2}+c_{y s}^{2}} \text { and } R=\sqrt{c_{x}^{2}+c_{y}^{2}} .
$$

6) Rotation-scale: In rotation-scale attack, $I\left(w_{I} \times h_{I}\right)$ is first rotated by an angle $\theta_{r s}$ and then scaled down to the original size. Let $I_{r s}$ be the attacked image. Following steps are executed in order to reverse this attack:
Step 1: Let $I_{g}$ be the Gaussian low pass filtered version of $I_{r s}$.

Step 2: Calculate the correction angle $\theta_{c}$ for $I_{g}$.

Step 3: Rotate $I_{g}$ by the angle $-\theta_{c}$. Let the rotated image be $I_{g c}$.

Step 4: Resize $I_{g c}$ to $\left(w_{I} \times h_{I}\right)$. Let the resized image be $I_{g c s}$.

Step 5: Find the IC $C_{g c s}=\left(c_{x g c s}, c_{y g c s}\right)$ of $I_{g c s}$ as in II.B.

Step 6: Find the IC $C_{g c}=\left(c_{x g c}, c_{y g c}\right)$ of $I_{g c}$ using $C_{g c s}$ and the scaling factor between $I_{g c}$ and $I_{g c s}$.

Step 7: Find the IC $C_{g}=\left(c_{x g}, c_{y g}\right)$ of $I_{g}$ by rotating the centroid $C_{g c}=\left(c_{x g c}, c_{y g c}\right)$ of $I_{g c}$ by the angle $\theta_{c}$.

Step 8: Let the linear distance of $C_{g}$ from the center of $I_{g}$ be $R_{g}$ and that of $C$ from the center of $I$ be $R$.

Step 9: Find the scaling factor $s_{r s}=R_{g} / R$.

Step 10: Resize $I_{g}$ using the scaling factor $1 / s_{r s}$. Let this resized image be $I_{g s}$.

Step 11: Crop $I_{g s}$ to the original size. Let this resized image be $I_{\text {gscp }}$ which is the cropped rotated image of the original image.

Step 12: Find the IC $C_{g s c p}=\left(c_{x g s c p}, c_{y g s c p}\right)$ of $I_{g s c p}$ as in II.B.

Step 13: Calculate the rotation angle $\theta_{r s}$, between $C$ and $C_{g s c p}$.

Step 14: First resize the input image $I_{r s}$ by the factor $1 / s_{r s}$ and then rotate by the angle $-\theta_{r s}$. Let this scaled and rotated image be $I_{r s}^{\prime}$ which is the restored approximation of the original image $I$.

\section{Other Combination of Attacks}

In the next section while restoring to the approximate original, it is assumed that a flipping attack follows other attacks. However, if other attacks follow a flipping attack their reversing processes may need to be updated. If a translation attack follows a flipping attack, we need to update (6) as

$$
t_{x}=c_{x t} \mp c_{x} \text { and } t_{y}=c_{y t}-c_{y}
$$

for a flipping around $y$-axis and

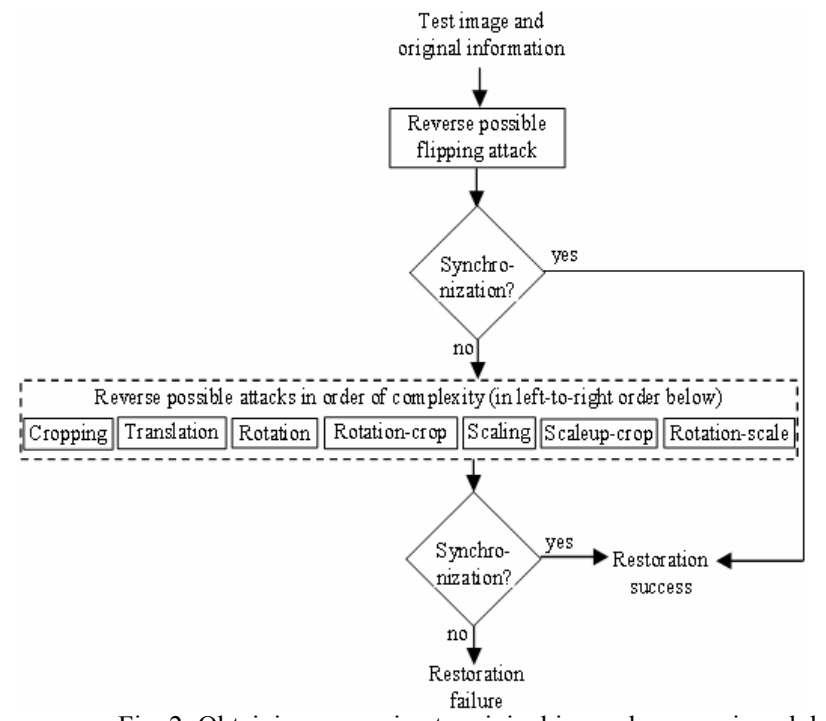

Fig. 2: Obtaining approximate original image by reversing global geometric attacks 


$$
t_{x}=c_{x t}-c_{x} \text { and } t_{y}=c_{y t} \mp c_{y}
$$

for a flipping around $x$-axis. If a rotation attack follows any flipping attack, then we need to update (7) as

$$
\theta_{r}= \pm\left(\alpha_{r}-\alpha\right)
$$

But if a cropping or a scaling attack follows any flipping attack, we do not need to update. Therefore, only the reversing processes of translation, rotation, rotation-crop, and rotationscale, discussed in section II.C above, are required to be updated accordingly. To reverse other global geometric transformations e.g., aspect-ratio change and any other possible combinations is currently under investigation. In the next section, a watermarking synchronization step, in which the extracted watermark is compared with the original watermark, is assumed.

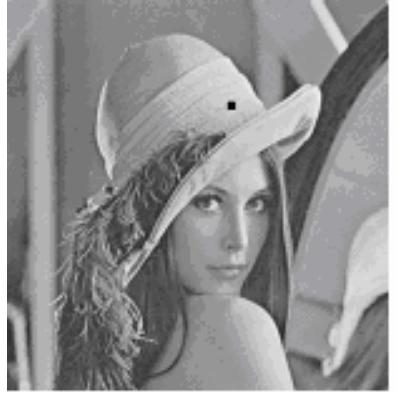

(a)

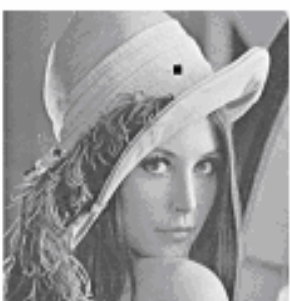

(b)
Fig. 3: (a) Original image $(512 \times 512)$ and (b) $25 \%$ cropped image $(384 \times 384)$; invariant centroids are shown as black dots

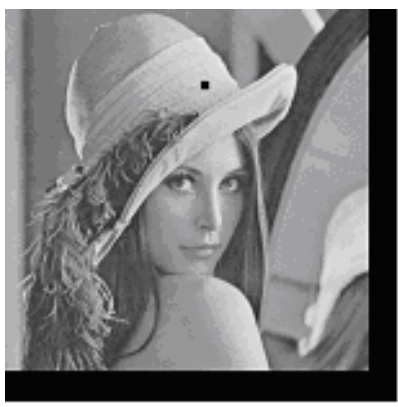

(a)

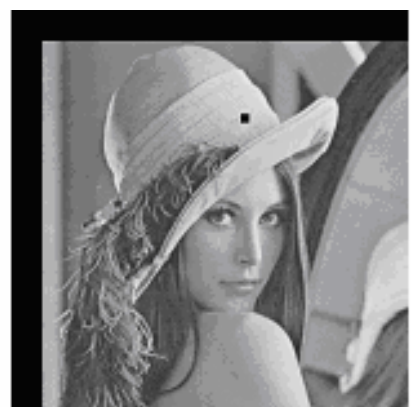

(b)
Fig. 4: Reversing translation: (a) translated image to $(-40,40)$ and

(b) translation restored image, estimated translation was $(-40,40)$; invariant centroids are shown as black dots

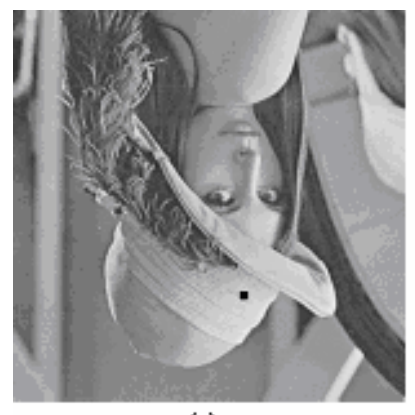

(a)

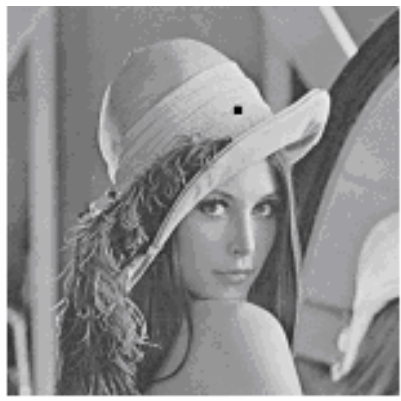

(b)
Fig. 5: Reversing flipping: (a) flipped (around $x$-axis) image and (b) flip restored image ( $\tilde{\mu}_{0,1}$ was positive for original image and negative for flipped image); invariant centroids are shown as black

\section{E. Obtaining Approximate Original}

Fig. 2 shows the restoration procedure to obtain the approximate original image by reversing global geometric transformations. The test image along with the required original information e.g., $w_{I}, h_{I}, C, r$, and signs of $\tilde{\mu}_{1,0}$ and $\tilde{\mu}_{0,1}$ are given as inputs. The test image is first inspected for a possible flipping attack that might follow other attacks. If the test image causes synchronization failure after reversing flipping attack, it is then inspected for attacks like cropping, translation, rotation, rotation-crop, scale, and rotation-scale sequentially. After reversing a possible attack (e.g., cropping) if it still causes synchronization failure, it is further inspected for the next attack (e.g., translation). The inspection is executed in ascending order of complexities of reversing processes. Once the tested image recovers synchronization after reversing some attack, the inspection terminates.

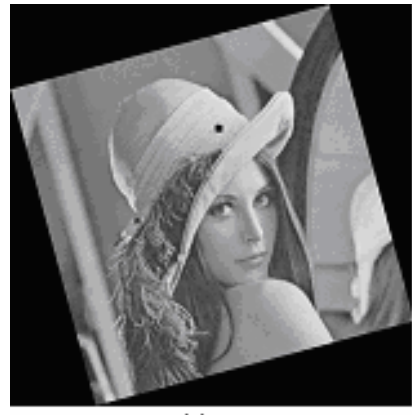

(a)

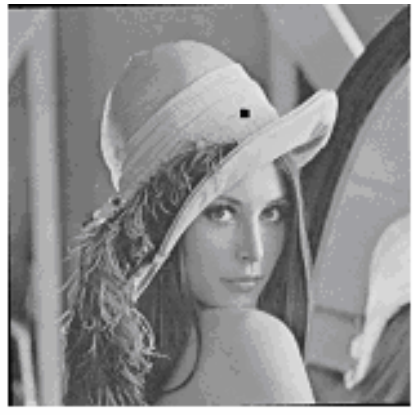

(b)
Fig. 6: Reversing rotation: (a) $15^{\circ}$ rotated image $(629 \times 629)$ and (b) rotation restored image $\left(512 \times 512\right.$, estimated angle was $\left.14.35^{\circ}\right)$; invariant centroids are shown as black dots

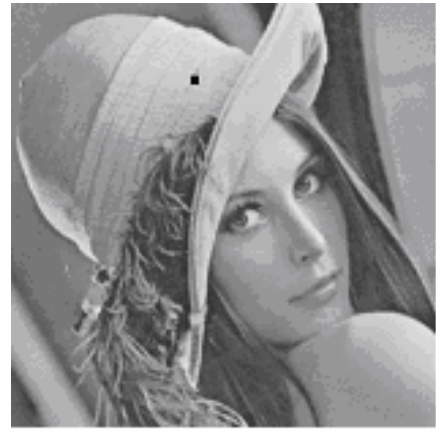

(i)

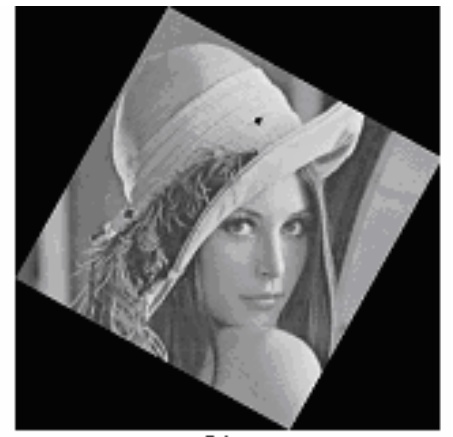

(b)
Fig. 7: Reversing rotation-crop: (a) $30^{\circ}$ rotated and cropped image $(374 \times 374)$ and $(b)$ restored image $(509 \times 509$, estimated angle was $29.14^{\circ}$ ): invariant centroids are shown as black dots

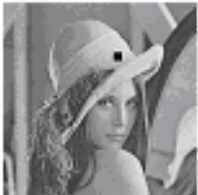

(a)

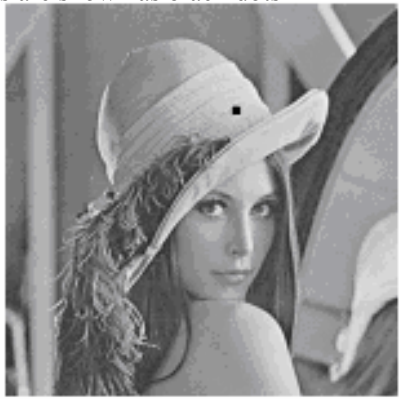

(b)
Fig. 8: Reversing scaling: (a) 50\% scaled $(256 \times 256)$ image and (b) restored image $\left(510 \times 510\right.$, estimated rotation angle was $0^{\circ}$ and scaling factor was 0.502); invariant centroids are shown as black dots 


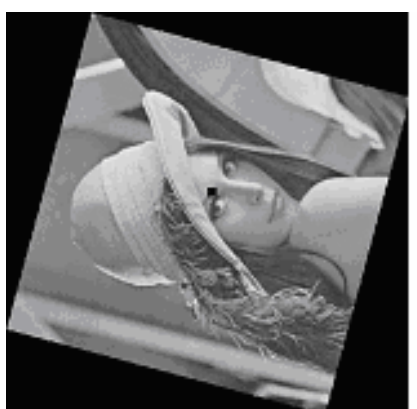

(a)

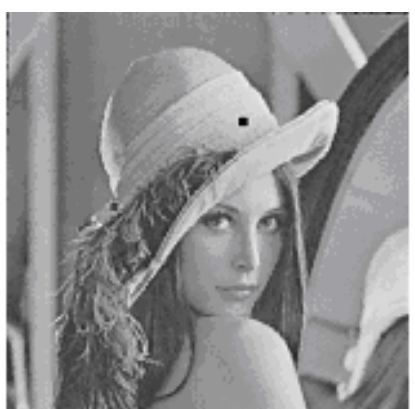

(b)
Fig. 9: Reversing rotation-scale: (a) $75^{\circ}$ rotated and $81 \%$ scaled image (512

$\times 512$, wrong invariant centroid was found) and (b) restored image $(512 \times$

512 , estimated scaling factor was 0.813 and rotation angle was $\left.75.52^{\circ}\right)$; invariant centroids are shown as black dots

\section{EXPERIMENTAL RESULTS}

We implemented the proposed scheme with MATLAB 7 and tested its effectiveness using many images. Due to space limitations, however, only results obtained using 8-bit grayscale Lena image $(512 \times 512)$ are presented. The radius of circular region while finding the IC was $r=64$. The IC was found at $C=(44,116)$ in the original Lena image, as shown in Fig. 3(a); hence, its angular distance was $\alpha=69.23^{\circ}$.

In $25 \%$ cropping attack, the IC was obtained accurately at $(44,116)$, as shown in Fig. 3(b), and no restoration was required. In translation attack, the origin $\left(x_{0}, y_{0}\right)=(0,0)$ was translated to $(-40,40)$ and the IC was found at $C_{t}=(4,156)$

TABLE I. EXPERIMENTAL RESULTS BY THE PROPOSED SCHEME AGAINST VARIOUS GEOMETRIC ATTACKS USING LENA IMAGE $(512 \times 512)$

\begin{tabular}{|c|c|c|c|c|}
\hline \multirow{2}{*}{ Attacks } & \multicolumn{2}{|c|}{ PSNR (dB) } & \multicolumn{2}{|c|}{ Estimated } \\
\hline & Before restore & After restore & Angle ( ${ }^{\circ}$ & Scale-factor \\
\hline Cropping $(25 \%, 384 \times 384)$ & $--^{b}$ & $--^{b}$ & 0.00 & 1.00 \\
\hline Flip $x$ (around $x$-axis) ${ }^{\mathrm{a}}$ & 13.57 & $\infty$ & $--^{c}$ & $--^{\mathrm{c}}$ \\
\hline Flipy (around $y$-axis) ${ }^{\mathrm{a}}$ & 12.49 & $\infty$ & $--^{c}$ & $--^{c}$ \\
\hline Flip $x$-rot. $\left(60^{\circ}, 701 \times 701\right)$ & $--^{b}$ & 23.95 & $-60.55^{d}$ & $--^{\mathrm{c}}$ \\
\hline Flipy-rot. $\left(75^{\circ}, 699 \times 699\right)$ & $--^{b}$ & 23.24 & $-75.72^{d}$ & $--^{\mathrm{c}}$ \\
\hline Flip $x$-rot.-autocrop $\left(75^{\circ}\right)^{a}$ & 11.01 & 15.07 & $-75.32^{d}$ & $--^{c}$ \\
\hline Flipy-rot.-autocrop $\left(90^{\circ}\right)^{\mathrm{a}}$ & 12.35 & 26.11 & $-90.15^{d}$ & $--^{\mathrm{c}}$ \\
\hline Flip $x$-rot.-scale $\left(45^{\circ}, 0.70\right)^{\mathrm{a}}$ & 7.32 & 28.52 & $-45.07^{d}$ & 0.752 \\
\hline Flipy-rot.-scale $\left(60^{\circ}, 0.73\right)^{\mathrm{a}}$ & 7.58 & 27.58 & $-60.00^{\mathrm{d}}$ & 0.775 \\
\hline Flip $x$-Translation $(-30,30)$ & 10.49 & 14.95 & $--^{\mathrm{c}}$ & $--^{c}$ \\
\hline Flipy-Translation $(-30,30)$ & 11.14 & 14.95 & $--^{c}$ & $--^{c}$ \\
\hline Rotation $\left(15^{\circ}, 699 \times 699\right)$ & $--^{b}$ & 20.93 & 14.35 & $--^{c}$ \\
\hline Rotation $\left(90^{\circ}, 512 \times 512\right)$ & 12.35 & 26.11 & 90.00 & $--^{\mathrm{c}}$ \\
\hline Rot.-autocrop-flip $x\left(45^{\circ}\right)^{\mathrm{a}}$ & 10.61 & 12.81 & 44.20 & $--^{c}$ \\
\hline Rot.-autocrop-flipy $\left(60^{\circ}\right)^{\mathrm{a}}$ & 10.43 & 13.35 & 60.05 & $--^{c}$ \\
\hline Rot.-crop $\left(15^{\circ}, 512 \times 512\right)$ & 11.93 & 14.58 & 14.44 & $--^{\mathrm{c}}$ \\
\hline Rot.-crop $\left(30^{\circ}, 374 \times 374\right)$ & $--^{b}$ & $--^{b}$ & 29.14 & $--^{c}$ \\
\hline Rot.-flip $x\left(30^{\circ}, 701 \times 701\right)$ & $--^{b}$ & 18.36 & 28.24 & $--^{c}$ \\
\hline Rot.-flipy $\left(45^{\circ}, 725 \times 725\right)$ & $--^{b}$ & 23.85 & 44.22 & $--^{\mathrm{c}}$ \\
\hline Rotation-scale $\left(75^{\circ}, 0.81\right)^{\mathrm{a}}$ & 8.43 & 24.93 & 75.52 & 0.813 \\
\hline Rotation-scale $\left(90^{\circ}, 1.00\right)^{\mathrm{a}}$ & 12.35 & 17.93 & 90.36 & 0.952 \\
\hline Rot.-scale-flip $x\left(15^{\circ}, 0.81\right)^{\mathrm{a}}$ & 8.64 & 19.90 & 14.47 & 0.787 \\
\hline Rot.-scale-flipy $\left(30^{\circ}, 0.73\right)^{\mathrm{a}}$ & 7.58 & 22.67 & 29.24 & 0.719 \\
\hline Scale $(0.50,256 \times 256)$ & $--^{b}$ & $--^{b}$ & $--^{\mathrm{c}}$ & 0.502 \\
\hline Scaleup-crop $(2.00,700 \times 800)$ & $--^{b}$ & $--^{b}$ & $--^{c}$ & 2.043 \\
\hline Translation $(-40,40)$ & 10.32 & 13.54 & $--^{c}$ & $--^{c}$ \\
\hline Translation-flip $x(-50,40)$ & 9.78 & 12.76 & $--^{c}$ & $--^{\mathrm{c}}$ \\
\hline Translation-flip $y(-50,40)$ & 10.20 & 12.76 & $--^{\mathrm{c}}$ & $--^{\mathrm{c}}$ \\
\hline
\end{tabular}

${ }^{\mathrm{a}} 512 \times 512 \mathrm{size} ;{ }^{\mathrm{b}}$ Not measured due to unmatched sizes; ${ }^{\mathrm{c}}$ Not necessary to measure; ${ }^{\mathrm{d}}$ flipping was reveresed first, threfore angles were found negated

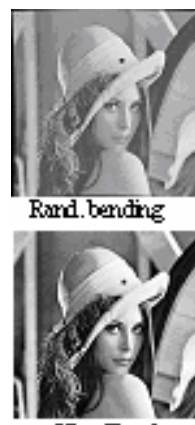

Hist. Equl

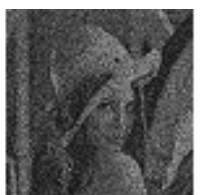

Hoise $20 \%$

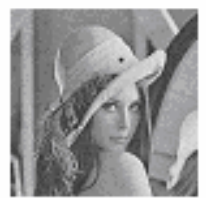

Grussimnoise

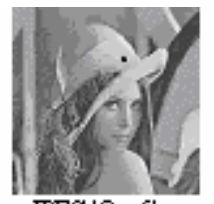

TPEG(Q= 5)

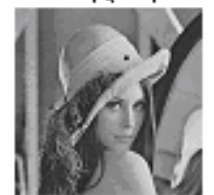

BPM

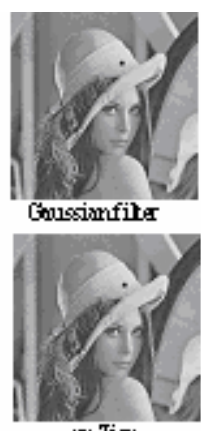

urigr
Fig. 10: Invariant centroid in local geometric and waveform attacks

for the attacked image; hence the estimated translation was the same $C_{t}-C=(-40,40)$. The attacked and restored images are shown in Fig. 4(a)-(b).

In flipping around the $x$-axis, the IC was found at (44,116); however, the sign of $\widetilde{\mu}_{0,1}$ was positive and negative for the original and attacked images respectively. Therefore, the attacked image was flipped around the $x$-axis in order to restore to the original image, as shown in Fig. 5(a)-(b). After restoration we found the IC accurately at $(44,116)$.

In $15^{\circ}$ rotation-only attack shown in Fig. 6(a), the angular distance of the IC was $\alpha_{r}=83.58^{\circ}$. Consequently, the estimated rotation angle became $\theta_{r}=\alpha_{r}-\alpha=14.35^{\circ}$. The attacked image was rotated by $-14.35^{\circ}$ and then cropped to the original size $(512 \times 512)$. The IC was found at $(45,115)$ in the restored image as shown in Fig. 6(b).

In the case of $30^{\circ}$ rotation-crop attack shown in Fig. 7(a), the angular distance of the IC was $\alpha_{r}=98.37^{\circ}$. Consequently, the estimated rotation angle was $\theta_{r}=\alpha_{r}-\alpha=29.14^{\circ}$. The attacked image was rotated by $-29.14^{\circ}$. The IC was found at $(45,115)$ in the restored image as shown in Fig. 7(b).

In $50 \%$ scaling attack shown in Fig. 8(a), the estimated scaling factor was found as $s=0.502$; so the restored image size was $510 \times 510(256 / \mathrm{s}=510)$. The IC was found at $(47,113)$ in the restored image as shown in Fig. 8(b).

In $75^{\circ}$ rotation followed by $81 \%$ scaling attack, the IC was found at a wrong position as shown in Fig. 9(a). The estimated scaling factor was found as $s_{r s}=0.813$ and the estimated rotation angle became $\theta_{r s}=75.52^{\circ}$. After a scaling by the factor $1.23\left(1 / s_{r s}\right)$ followed by a rotation by $-75.52^{\circ}$, it was then cropped to the original size. The IC was found at $(45,116)$ in the restored image shown in Fig. 9(b).

TABLE I summarizes some experimental results, including above, against various global geometric attacks using Lena image. We observed that the proposed scheme reverses global geometric transformations with very high accuracy. TABLE II compares the robustness of the proposed content-based scheme with existing content-based schemes against global geometric attacks. The comparison is according to the results what the authors claimed in their papers. The proposed scheme is robust to rotation-scale, flipping and many other attacks, as shown in TABLE I, to which existing schemes are vulnerable. However, like other content-based schemes it is not robust to aspect-ratio change due to possible sensitivity of the IC to this attack. The image normalization- 
TABLE II. COMPARISONS OF THE ROBUSTNESS OF CONTENT-BASED SCHEMES AGAISNT GLOBAL GEOTERIC TRANSFORMATIONS

\begin{tabular}{|c|c|c|c|c|c|c|c|c|c|}
\hline Schemes & Using & Rotation & $\begin{array}{c}\begin{array}{c}\text { Rotation- } \\
\text { crop }\end{array} \\
\end{array}$ & $\begin{array}{c}\text { Rotation- } \\
\text { scale }\end{array}$ & Cropping & Scaling & $\begin{array}{c}\begin{array}{c}\text { Transla- } \\
\text { tion }\end{array} \\
\end{array}$ & Flipping & $\begin{array}{c}\begin{array}{c}\text { Aspect- } \\
\text { ratio }\end{array} \\
\end{array}$ \\
\hline Bas et al. [9] & Harris detector & yes & no & no & yes & yes & yes & no & no \\
\hline Alghoniemy and Tewfik [14] & wavelet maxima & yes & no & yes & yes & yes & no & no & no \\
\hline Tang and Hang [15] & Mexican Hat wavelet & no & yes & no & yes & no & yes & no & no \\
\hline Lee et al. [16] & scale-invariant features & yes & no & no & yes & yes & yes & no & no \\
\hline Nikolaidis and Pitas [17] $^{\mathrm{a}}$ & image segmentation & no & yes & no & yes & yes & no & no & no \\
\hline Seo and Yoo [18] & scale-space & no & yes & no & yes & yes & no & no & no \\
\hline Qi and Qi [19] & improved Harris detector & no & yes & yes & yes & yes & yes & no & no \\
\hline Feng and Izquierdo [20] & Harris detector and curvature scale-space & yes & no & no & no & no & no & no & no \\
\hline Proposed & invariant centroid and geometric moments & yes & yes & yes & yes & yes & yes & yes & no \\
\hline
\end{tabular}

${ }^{\text {a }}$ robust to small rotation angles and upscaling only, but severely weak to downscaling

based schemes in [10] and [11] were robust against small aspect ratio change. However, they were highly sensitive to cropping. Moreover, the IC also remained constant in local geometric attacks like random bending attacks and waveform attacks like JPEG and noising, as shown in Fig. 10.

\section{CONCLUSIONS}

The proposed geometric distortion correction scheme can restore a global geometric attacked image to its approximate original image with very high accuracy by reversing the operation. We can obtain $100 \%$ accuracy by combining the proposed scheme with an exhaustive search scheme [1], where only a few iterations will be required. The proposed scheme can successfully resist many global geometric transformations, including flipping and rotation-scale (and more in TABLE I) attacks to which many of the existing schemes were vulnerable. Its low computational complexity, due to use of first and second order geometric moments only, enables real time applications. We hope it would be exploited effectively for copyright protection of digital images and videos. The watermark embedding area could be specified with respect to the IC for easing the synchronization.

But the reference point IC was found sensitive against aspect-ratio change and high up and down scaling. This is because interpolation changes the content of the image region used to calculate it. Moreover, some original information was assumed to be available in the detection phase. For practical implementation, we suggest to dedicate a few bytes of the watermarking secret key to pass this information, other than the image size on which both encoder and decoder can agree beforehand. It is worth mentioning that the IC is highly dependant on the value of $r$. While there are a few of such points for large $r$, there are many of them for small $r$. However, such reference point is sensitive to cropping when $r$ is large. Delannay and Macq [23] noted the same problem for their reference system. Therefore, the image boundary area is not considered while finding a large $r$ that offers minimum number of reference points. Ideally only one reference point is expected which in turn voids its sensitivity to initial value. Future works includes how to get rid of all above problems.

\section{REFERENCES}

[1] Alghoniemy, M and A. H. Tewfik, "Progressive quantized projection watermarking scheme," in Proc. ACM Multimedia Conference, pp. 295-298, 1999.

[2] Hartung, F., J. K. Su, and B. Girod, "Spread spectrum watermarking: malicious attacks and counter-attacks," in Proc. Security and Watermarking of Multimedia Contents I, vol. SPIE 3657, pp. 147-158, 1999.
[3] Pereira, S. and T. Pun, "Fast robust template matching for affine resistant image watermarks," in Proc. of International Workshop on Information Hiding, vol. LNCS 1768, pp. 200-210, 1999.

[4] Voloshynovskiy, S., A. Herrigel, and Y. B. Rytsar, "Watermark template attack," in Proc. Security Watermarking of and Multimedia Contents III, vol. SPIE 4314, pp. 394 405, 2001.

[5] Ruanaidh, J. J. K. Ó and T. Pun, "Rotation, scale and translation invariant spread spectrum digital image watermarking," Signal Processing, vol. 66(3), pp. 303-317, 1998

[6] Lin, C. Y., J. A. Bloom, I. J. Cox, M. Miller, and Y. Lui, "Rotation-, scale-, and translationresilient public watermarking of images," in Proc. Security and Watermarking of Multimedia Contents II, vol. SPIE 3971, pp. 90-98, 2000.

[7] Kim, B. S., J. G. Choi, C. H. Park, J. U. Won, D. M. Kwak, S. K. Oh, C. R. Koh, and K. H. Park, "Robust digital image watermarking method against geometrical attacks," Real Time Imaging, vol. 9(2), pp. 139-149, 2003.

[8] Dong, P., J. G. Brankov, and N. P. Galatsanos, "Geometric robust watermarking based on a new mesh model correction approach," in Proc. International Conference on Image Processing, vol. 3, pp. 493-496, 2002.

[9] Bas, P., J. M. Chassery, and B. Macq, "Geometrically invariant watermarking using feature points," IEEE Transactions on Image Processing, vol. 11(9), pp. 1014-1028, 2002.

[10] Dong, P., J. B. G. Brankov, N. P. Galatsanos, Y. Yang, and F. Davoine, "Digital watermarking robust to geometric distortions," IEEE Transactions on Image Processing, vol. 14(12), pp. 2140-2150, 2005

[11] Alghoniemy, M and A. H. Tewfik, "Geometric invariance in image watermarking," IEEE Transactions on Image Processing, vol. 13(2), pp. 145-153, 2004.

[12] Kim, H. S and H. K. Lee, "Invariant image watermark using zernike moments," IEEE Transactions on Circuits and Systems for Video Technology, vol. 13(8), pp. 766-775, 2003.

[13] Kutter, M., S. K. Bhattacharjee, and T. Ebrahimi, "Towards second generation watermarking schemes," in Proc. International Conference on Image Processing, vol. 1, pp. 320-323, 1999.

[14] Alghoniemy, M and A. H. Tewfik, "Progressive quantized projection approach to data hiding," IEEE Transactions on Image Processing, vol. 15(2), pp. 459-472, 2006.

[15] Tang, C. W. and H. M. Hang, "A feature-based robust digital image watermarking scheme," IEEE Transactions on Signal Processing, vol. 51(4), pp. 950-959, 2003.

[16] Lee, H. Y., J. T. Kim, H. K. Lee, and Y. H. Suh, "Content-based synchronization using the local invariant feature for robust watermarking," in Proc. International Workshop on Information Security Applications, vol. LNCS 3325, pp. 122-134, 2004.

[17] Nikolaidis, A. and I. Pitas, "Region-based image watermarking," IEEE Transactions on Image Processing, vol. 10(11), pp. 1726-1740, 2001.

[18] Seo, J. S. and C. D. Yoo, "Localized image watermarking based on feature points of scale-space representation," Pattern Recognition Letters, vol. 37(7), pp. 1365-1375, 2004.

[19] Qi, X. and J. Qi, "Image content-based geometric transformation resistant watermarking approach," in Proc. International Conference on Accoustics, Speech, and Signal Processing, vol. 2, pp. 829-832, 2005.

[20] Feng, Y. and E. Izquierdo, "Robust local watermarking on salient image areas," in Proc. International Workshop on Digial Watermarking, vol. LNCS 2613, pp. 189-201, 2003.

[21] Celik, M. U., E. Saber, G. Sharma, and A. M. Tekalp, "Analysis of feature-based geometry invariant watermarking," in Proc. Security and Watermarking of Multimedia Contents III, vol. SPIE 4314, pp. 261-268, 2001.

[22] Barni, M., "Effectiveness of exhaustive search and teplate matching against watermark desynchronization," IEEE Signal Processing Letters, vol. 12(2), pp. 158-161, 2005.

[23] Delannay, D. and B. Macq, "Watermarking relying on cover signal content to hide synchronization marks," IEEE Transactions on Information Forensics and Security, vol. 1(1), pp. 87-101, 2006. 